\title{
PROGRESS IN THE DEVELOPMENT OF A CLASS OF EFFICIENT LOW DISSIPATIVE HIGH ORDER SHOCK-CAPTURING METHODS ${ }^{1}$
}

\author{
H.C. Yee $^{2}$, B. Sjogreen ${ }^{3}$, N.D. Sandham ${ }^{4}$ and A. Hadjadj ${ }^{5}$
}

Proceedings of the Symposium in Computational Fluid Dynamics for the 21st Century July 15-17, 2000, Kyoto, Japan

and

RIACS Technical Report 00.11

June 2000, NASA Ames Research Center

\footnotetext{
${ }^{1}$ Partial of this work was carried out while the second, third and fourth authors were visiting scientists with RIACS. NASA Ames Research Center

${ }^{2}$ NASA Ames Research Center, Moffett Field, CA 94035, USA

${ }^{3}$ Department of Numerical Analysis and Computer Science, KTH, 10044 Stockholm, Sweden

${ }^{4}$ School of Engineering Sciences, University of Southampton, Southampton SO17 1BJ, UK

${ }^{5}$ LMFN-CORIA-UMR CNRS 6614, INSA du Madrillet 76801 St Etienne du Rouvray, France
} 


\title{
PROGRESS IN THE DEVELOPMENT OF A CLASS OF EFFICIENT LOW DISSIPATIVE HIGH ORDER SHOCK-CAPTURING METHODS ${ }^{1}$
}

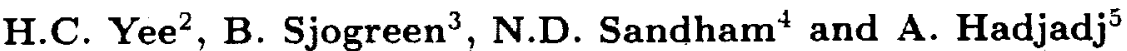

\begin{abstract}
In a series of papers, Olsson (1994, 1995), Olsson \& Oliger (1994), Strand (1994), Gerritsen \& Olsson (1996), Yee et al. (1999a,b, 2000) and Sandham \& Yee (2000), the issue of nonlinear stability of the compressible Euler and Navier-Stokes Equations, including physical boundaries, and the corresponding development of the discrete analogue of nonlinear stable high order schemes, including boundary schemes, were developed, extended and evaluated for various fluid flows. High order here refers to spatial schemes that are essentially fourth-order or higher away from shock and shear regions. The objective of this paper is to give an overview of the progress of the low dissipative high order shock-capturing schemes proposed by Yee et al. (1999a,b, 2000). This class of schemes consists of simple non-dissipative high order compact or noncompact central spatial differencings and adaptive nonlinear numerical dissipation operators to minimize the use of numerical dissipation. The amount of numerical dissipation is further minimized by applying the scheme to the entropy splitting form of the inviscid flux derivatives, and by rewriting the viscous terms to minimize odd-even decoupling before the application of the central scheme (Sandham \& Yee).

The efficiency and accuracy of these schemes are compared with spectral, TVD and fifthorder WENO schemes. A new approach of Sjogreen \& Yee (2000) utilizing non-orthogonal multi-resolution wavelet basis functions as sensors to dynamically determine the appropriate amount of numerical dissipation to be added to the non-dissipative high order spatial scheme at each grid point will be discussed. Numerical experiments of long time integration of smooth flows, shock-turbulence interactions, direct numerical simulations of a 3-D compressible turbulent plane channel flow, and various mixing layer problems indicate that these schemes are especially suitable for practical complex problems in nonlinear aeroacoustics, rotorcraft dynamics, direct numerical simulation or large eddy simulation of compressible turbulent flows at various speeds including high-speed shock-turbulence interactions, and general long time wave propagation problems. These schemes, including entropy splitting, have also been extended to freestream preserving schemes on curvilinear moving grids for a thermally perfect gas (Vinokur \& Yee 2000).
\end{abstract}

\section{Motivation and Overview}

Strong theoretically based high order high-resolution shock-capturing schemes have dominated algorithm development for fluid flows for nearly two decades. During the 1990's, the

\footnotetext{
${ }^{1}$ Proceedings of the Symposium in Computational Fluid Dynamics for the 21st Century, July 15-17, 2000, Kyoto, Japan; RIACS Technical Report 00.11, June 2000, NASA Ames Research Center; Partial of this work was carried out while the second. third and fourth authors were visiting scientists with RIACS, NASA Ames Research Center

2NASA Ames Research Center. Moffett Field, CA 94035, USA

${ }^{3}$ Department of Numerical Analysis and Computer Science, KTH, 10044 Stockholm, Sweden

${ }^{4}$ School of Engineering Sciences, University of Southampton, Southampton SO17 1BJ, UK

'LMFN-CORIA-UMR CNRS 6614, INSA du Madrillet 76801 St Etienne du Rouvray, France
} 
focus was on increasing the order of accuracy for the interior grid points of the computational domain with stable numerical boundary conditions generally not included as part of the theoretical development. Multidimensional upwinding and high order unstructured or finite element constructions have flooded the computational fluid dynamics (CFD) literature. The majority of these schemes are either too CPU intensive for practical applications or they are still in the development stage. In spite of their high-resolution capability for rapidly evolving flows and short time integrations, these schemes often exhibit undesirable amplitude errors for long time integrations. On the other hand, simplicity, efficiency and highly parallelizable robust algorithms are the major requirements in industrial, geophysical, space exploration and military practical CFD applications. The objective of this paper is to give an overview of the progress of a class of simple. highly parallelizable high order shock-capturing schemes that meets many of the requirements for practical computations, especially for long time integrations of unsteady flows. For ease of reference, "schemes" or more precisely "interior schemes" here generally refer to spatial difference schemes for the interior grid points of the computational domain, whereas "boundary schemes" are the numerical boundary difference operators for grid points near the boundaries. However, without loss of generality, we also adopt the conventional terminology of denoting "scheme" as either the "combined interior and boundary schemes" or just the "interior scheme" interchangeably within the context of the discussion.

Before 1994. rigorous stability estimates for accurate and appropriate boundary schemes associated with fourth-order or higher spatial interior schemes were the major stumbling block in the theoretical development of combined interior and boundary schemes for nonlinear systems of conservation laws. Spatial nonlinear stability of initial boundary value problems (IBVPs) goes hand-in-hand with the appropriate amount of nonlinear numerical dissipation required to stabilize the combined interior and boundary schemes. The delicate balance of the numerical dissipation for stability without the expense of excessive smearing of the flow physics after long time integrations poses a severe challenge for unsteady flow simulations of this type. Actually, there are two possible sources of stabilizing mechanisms; namely, (a) from the governing equation level and (b) from the numerical scheme level. Employing a nonlinear stable form of the governing equations (more conditioned form of the $\mathrm{PDE}$ ) in conjunction with the appropriate nonlinear stable scheme for IBVPS is one way of minimizing the use of numerical dissipation.

The major tool used to overcome the stumbling block is a generalized energy method. The basic building block in establishing a stable energy estimate for high order spatial central schemes for nonlinear hyperbolic conservation laws relies on the aforementioned mechanisms (a) and (b). From the governing equation level, a special transformation of the conservation laws to an appropriate form for the application of the continuous energy estimate for a stable IBVP of the governing equations is needed. From the numerical scheme level, a compatible boundary scheme for high orler central interior schemes that satisfy the discrete analogue of the contimous energy estimate is needed. See Strand (1994), Olsson (1995). Olsson \& Oliger (1994) and references cited.

Olsson proved that an energy estimate can be established for second-order central schemes. To obtain a rigorous energy estimate for high order central schemes, one must apply the scheme to the split form of the inviscid governing equation. For the Euler equations, the transformation consists of a convex entropy function that satisfies a mathematical entropy condition. This mathematical entropy function, in this case, can be a function of the physical entropy. Therefore, the resulting splitting is hereafter referred to as entropy splitting for ease of reference. Here, the entropy splitting should not be confused with the traditional flux vector splittings such as the Steger and Warming splitting (1981) or other variants. The traditional flux vector splitting splits the flux function into different parts and most often into upwind 
and downwind portions. However, the entropy splitting splits the flux derivatives and the time derivative of the conservative dependent variables using the properties of the chosen mathematical entropy function and the symmetrizability of the conservation laws, without reference to any upwinding. For the viscous terms, only symmetry is needed in the derivation of the energy estimate. These developments have made high order non-dissipative spatial central schemes of renewed interest to CFD practitioners where simplicity, efficiency and high parallelizability are their trademark.

The recently developed high order low-dissipation shock capturing schemes using characteristic filters of Yee et al. (1999a) fit in the entropy splitting framework. Instead of applying a scalar dissipation or filter (Gerritsen \& Olsson), they supply nonlinear filters based on, locally, the different wave characteristics of the inviscid flux. For complex shock waves, shear and turbulence interactions, one has better control of the amount of dissipation associated with each wave. For efficiency, Yee et al. proposed a combination of a high order base scheme and a nonlinear filter operator. The base scheme consists of narrow grid stencil high order compact or non-compact centered non-dissipative classical spatial differencings. The filter consists of a product of the dissipative portion of a low order total variation diminishing (TVD), essentially non-oscillatory (ENO) or weighted ENO (WENO) scheme and an artificial compression method (ACM) sensor. In contrast to hybrid schemes that switch between spectral or spectrallike non-shock-capturing schemes and high order ENO schemes, the high order non-dissipative base scheme is alwars activated. The role of the ACM sensor is to reduce the amount of numerical dissipation away from shock and shear regions. As an alternative to the ACM sensor, Sjogreen and Yee (2000) utilized non-orthogonal wavelet basis functions as multi-resolution sensors to dynamically determine the amount of nonlinear numerical dissipation to be added at each grid point. The resulting sensor function is also readily usable for grid adaptation purposes. The final grid stencil of these schemes is five points in each spatial direction if second-order TVD schemes are used as filters, and seven points if second-order ENO schemes are used as filters for a fourth-order base scheme. Studies showed that higher accuracy was achieved with less CPU time and fewer grid points when compared with that of standard high order TVD. positive. ENO or WENO schemes. Table 1 shows the flow chart of the schemes.

The studies in Yee et al. (1999b, 2000) and Sandham \& Yee indicate that entropy splitting can improve the overall stability of the scheme, and that the amount of numerical dissipation, if needed. is less than for the unsplit approach. They view entropy splitting as a conditioned form of the original governing equations. Here, "condition the governing equation" is different from "preconditioning of the flow equations or their discretized counterparts" in convergence acceleration of time-marching to steady states. Their studies also indicate that entropy splitting alone can improve nonlinear stability even when one employs boundary schemes that do not satisfy the discrete generalized energy estimate. This stability property of the entropy splitting is valuable not just for the class of schemes in question, but can also be applied to other schemes commonly used in practical CFD applications. This emphasizes the fact that one should always try to apply numerical schemes to a more conditioned form of the governing equations.

Extension of these schemes to freestream preserving schemes for 3-D curvilinear moving grids for a thermally perfect gas is reported in Vinokur and Yee (2000). The main difficulty in the extension of high order schemes to curvilinear grids is the high order numerical evaluation of the geometric terms, arising from the coordinate transformation, to satisfy a coordinate-invariant freestream preservation condition. The question of the extendibility of the entropy splitting concept to other physical equations of state and evolutionary equation sets was examined in Yee et al. (1999b. 2000). Their study shows that the entropy splitting can be formally 
extended to a thermally perfect gas, with the internal energy being an arbitrary function of temperature. For non-equilibrium flows which consist of a mixture of different species, each obeying a thermally perfect gas law, extension of the splitting is problematic. While they were able to prove the symmetry and homogeneity properties required for the energy estimate, the degree of homogeneity can only be obtained by solving a system of nonlinear equations. In addition, to obtain the Jacobian of the transformation required inverting a non-sparse linear system. It would therefore be difficult to establish the positive definite condition in closed form. Consequently, the extension of the method to non-equilibrium flows is not practically feasible. If the homogeneity condition is not required, then one can use symmetry variables based on the physical entropy, as was shown by Chalot et al. (1990). In this case, the resulting PDEs are in pure non-conservative form and entropy splitting is no longer applicable. For magnetohydrodynamics (MHD), the magnetic field has to be added as a "conservative" variable. But the square of the magnetic field is one of the terms in the definition of the total energy. Thus, from dimensional arguments it is clear that one cannot obtain the homogeneity condition. A similar situation exists for the artificial compressibility method of treating incompressible flow. In the Maxwell equations, we have a linear system of hyperbolic equations that can easily be symmetrized. Thus Strand's numerical boundary operators are still valid, but entropy splitting is not needed. For non-equilibrium flows, if one solves the species and flow equations separately in a loosely-coupled manner, then the flow equations effectively satisfy a locally thermally perfect gas law and a "local" form of entropy splitting is applicable.

We would like to point out that although the formal extension of entropy splitting is limited to a thermally perfect gas, the numerical schemes themselves do not have this restriction. Consequently. the schemes discussed here are applicable to equilibrium real gas, nonequilibrium and the artificial compressibility method of treating incompressible flows, MHD and the Maxwell equations.

\section{Entropy Splitting and Numerical Methods}

This section reviews the entropy splitting formula for the 2-D compressible Euler equations for a perfect gas in Cartesian coordinates. Formulas for the corresponding 3-D case can be found in Appendix B of Yee et al. (1999b) and for curvilinear moving grids for a thermally perfect gas in Vinokur and Yee (2000). The mathematical theory is quite involved. Interested readers are referred to references cited. The Yee et al. (1999a) and Sjogreen \& Yee numerical methods used in conjunction with the entropy splitting are also summarized.

\subsection{Summary of Entropy Splitting for a Perfect Gas}

In vector notation the 2-D compressible time-dependent Euler equations in conservation form for an equilibrium real gas can be written, in Cartesian coordinates, as

$$
U_{t}+F_{x}+G_{y}=0
$$

where $U_{t}=\frac{\partial L^{-}}{\partial t} \cdot F_{x}=\frac{\partial F}{\partial x}$ and $G_{y}=\frac{\partial G}{\partial y}$ and the $U, F, G$, are vectors given by

$$
L^{-}=\left[\begin{array}{c}
\rho \\
\rho u \\
\rho v \\
e
\end{array}\right]: \quad F=\left[\begin{array}{c}
\rho u \\
\rho u^{2}+p \\
\rho u v \\
e u+p u
\end{array}\right]: \quad G=\left[\begin{array}{c}
\rho v \\
\rho u v \\
\rho v^{2}+p \\
e v+p v
\end{array}\right]
$$


The dependent variable $U$ is the vector of conservative variables, and $(\rho, u, v, p)^{T}$ is the vector of primitive variables. Here $\rho$ is the density, $u$ and $v$ are the velocity components, $\rho u$ and $\rho v$ are the $x$ - and $y$-components of the momentum per unit volume, $p$ is the pressure, $e=$ $\rho\left[\varepsilon+\left(u^{2}+v^{2}\right) / 2\right]$ is the total energy per unit volume, and $\varepsilon$ is the specific internal energy. For a thermally perfect gas, the equation of state is $p=\rho R T$, where $R$ is the specific gas constant, and $T$ is the temperature with $\varepsilon=\varepsilon(T)$. For constant specific heats (calorically perfect gas) $\varepsilon=c_{v} T$, where $c_{v}$ is the specific heat at constant volume.

The eigenvalues associated with the flux Jacobian matrices of $F$ and $G$ are $(u, u, u \pm c)$ and $(v, v, v \pm c)$, where $c$ is the sound speed. The two $u, u$ and $v, v$ characteristics are linearly degenerate. Hereafter, we refer to the fields associated with the $u \pm c$ and $v \pm c$ characteristics as the nonlinear fields and the fields associated with the $u, u$ and $v, v$ characteristics as the linear fields.

The entropy splitting for the compressible Euler equations for a perfect gas utilizes the result of Harten (1983) on symmetric form for systems of conservation laws as the backbone. The idea is to introduce a symmetry transformation from the vector of conservative variables $U$ to a new vector $W$, referred to as the "entropy variables". The transformation is chosen so that $F_{W}=\frac{\partial F}{\partial W}$ and $G_{W}=\frac{\partial G}{\partial W}$ are symmetric, and $U_{W}=\frac{\partial U}{\partial W}$ is symmetric and positive definite. One then restricts the transformations to those that allow a special splitting of $F_{x}, G_{y}$ and $U_{t}$. This requires that the entropy variable $W$ be chosen such that $F(U(W)), G(U(W))$ and $U(W)$ are homogeneous functions of $W$ of degree $\beta$. Then the splitting of $F_{x}$ results in

$$
F_{x}=\frac{1}{3+1}\left(F_{W} W\right)_{r}+\frac{1}{\beta+1} F_{W} \cdot W_{x}=\frac{\beta}{\beta+1} F_{x}+\frac{1}{\beta+1} F_{W} W_{x}, \quad \beta \neq-1 .
$$

The corresponding $I F$ can be written as

$$
W^{-}=\frac{p^{*}}{p}[e-2 \rho \varepsilon-p(1+\beta) \quad-\rho u \quad-\rho v \quad \rho]^{T} ; \quad p^{*}=-\left(p \rho^{-\gamma}\right)^{\frac{1}{3(1-\gamma)}} .
$$

A similar splitting can be written for $G_{y}$ and $U_{t}$. The forms for $F_{\mathfrak{V}}$ and $G_{W}$ can be found in Yee et al. (1999b). Under the above conditions one can rigorously establish a bound on the rate of growth of the energy norm in terms of the eigenvalues corresponding to the incoming characteristic variables at the boundary of the domain.

Normally. we need to compute $U_{W}$ for the split form of $U_{t}=\frac{3}{3+1} U_{t}+\frac{1}{\beta+1} U_{W} W_{t}$. However, we only consider a semi-discrete approach of applying temporal discretizations. Aside from using the split form of the inviscid flux derivatives $F_{x}$ and $G_{y}$. we do not have to use the split form of $\tau_{t}^{-}$for implementation. Thus the final form of the semi-discrete entropy splitting approach still can be expressed in terms of conservative and primitive variables, making possible easy and efficient implementation in existing computer codes. The splitting parameter $\beta$ has to satisfy $\beta>0$ or $3<\frac{\gamma}{1-\gamma}$. Harten only considered $\beta<\frac{\gamma}{1-\gamma}$. This choice of $\beta$ appears to be "non-standard" or "nonphysical" in the sense that more than $100 \%$ of the conservative portion and a negative non-conservative portion is used. Although Gerritsen and Olsson considered the $\beta>0$ range which Harten overlooked, they set $\beta=1$ in conjunction with high order central differencing schemes in all of their numerical examples. This choice of $\beta$ corresponds to splitting of the flux derivative into equal conservative and non-conservative proportions. Yee et al. (1999b. 2000) recommend the use of $\beta>0$. The degree of improvement in stability over the unsplit approach depends on the choice of $\beta$. For $\beta>100$ the benefit from the entropy splitting is diminishing, since this case is close to the unsplit situation. In addition, the choice of $\beta$ is also problem-dependent. For certain problems, e.g., complex shock-shear interactions, 
the beneficial range of $\beta$ can be small. A combination of wavelets as filter (Sjogreen \& Yee) sensors and grid adaptations (Gerritsen \& Olsson) might be able to enlarge the beneficial range since the resulting wavelet sensor function is also readily applicable for grid adaptation purposes. The grid adaptation might be able to minimize the underresolved grid related spurious oscillation producing instability. This will be a subject of future research.

\subsection{Numerical Methods}

The spatial discretizations considered in Yee et al. (1999a) consist of two parts, namely, a base scheme and a filter. When numerical dissipations or filters are not used, the scheme consists of only the base scheme. If entropy splitting is used, the base scheme is applied to the split form of the inviscid flux derivatives. Possible non-dissipative high order base schemes for $F_{x}$ and $G_{y}$ and the viscous terms (if present) are the standard fourth and sixth-order compact and non-compact central schemes for the interior grid points.

There are many possible candidates for the filter operator in conjunction with high order base schemes. For efficiency and ease of numerical boundary treatment, Yee et al. (1999a) proposed using filter operators whose grid stencils have a width similar to that of the base scheme. The filter operator consists of the product of a sensor and a nonlinear dissipation. See Table 1 for the road map. Denote $F_{j, k}$ as the discrete approximation of the inviscid flux $F$ at $(j \Delta x, k \Delta y)$. where $\Delta x$ and $\Delta y$ are the grid spacings in the $x$-and $y$-directions and $j$ and $k$ are the corresponding spatial indices. Let the filter vector in the $x$-direction be of the form

$$
\tilde{F}_{j+\frac{1}{2}, k}^{*}=\frac{1}{2} R_{j+\frac{1}{2}} \Phi_{j+\frac{1}{2}}^{*}
$$

$\tilde{F}_{j+\frac{1}{2}, k}^{*}$ is the modified form of the nonlinear dissipation portion of the standard numerical flux. For characteristic based methods, the quantity $R_{j+\frac{1}{2}}$ (with the $k$ index suppressed) is the right eigenvector matrix of $\frac{\partial F}{\partial t}$ using Roe's average state (Roe's approximate Riemann solver). We define $\widetilde{G}_{j, k+\frac{1}{2}}^{*}$ in the same manner. The elements of $\Phi_{j+\frac{1}{2}}^{*}$ (with the $k$ index suppressed), denoted by $\left(\phi_{j+\frac{1}{2}}^{l}\right)^{*}$. are

$$
\left(\phi_{j+\frac{1}{2}}^{l}\right)^{*}=\mathcal{S}_{j+\frac{1}{2}}^{l} \phi_{j+\frac{1}{2}}^{l}
$$

$\phi_{j+\frac{1}{2}}^{l}$ in (5) is the dissipative portion of the high resolution scheme resulting from using a TVD, MUSCL with slope limiters. ENO or WENO scheme. Formulae for $\phi_{j+\frac{1}{2}}^{l}$ are well known and can be found in the literature. See Yee et al. (1999a) for details and for a discussion of other possible filters.

Here $\mathcal{S}_{j+\frac{1}{2}}^{l}$ is the sensor and is a mechanism for controlling excess numerical dissipation that is inherent in the dissipative portion of standard high-resolution shock-capturing schemes. Two possible sensors are considered. They are the ACM sensor and the wavelet sensor (Sjogreen \& Yee).

ACM Sensor: For the ACM filter, $\mathcal{S}_{j+\frac{1}{2}}^{l}=\kappa \theta_{j+\frac{1}{2}}^{l}$. The parameter $\kappa$ is problem-dependent. For smooth flows that are absent of high shears, $\kappa$ can be very small. It is used to minimize spurious high frequency oscillation producing nonlinear instability associated with pure central schemes, especially for long time integration problems. Different physical problems require different values of $k$ because of the large variation in flow properties. The $\kappa$ value may vary from one characteristic wave to another, and from one region of the flow field to another region 
with different flow structure. The function $\theta_{j+\frac{1}{2}}^{l}$ is the Harten ACM gradient sensor but utilized in a different context than Harten originally intended. For a general $2 m+1$ point base scheme, Harten recommended

$$
\begin{gathered}
\theta_{j+\frac{1}{2}}^{l}=\max \left(\hat{\theta}_{j-m+1}^{l}, \ldots, \hat{\theta}_{j+m}^{l}\right), \\
\hat{\theta}_{j}^{l}=\left|\frac{\left|\alpha_{j+\frac{1}{2}}^{l}\right|-\left|\alpha_{j-\frac{1}{2}}^{l}\right|}{\left|\alpha_{j+\frac{1}{2}}^{l}\right|+\left|\alpha_{j-\frac{1}{2}}^{l}\right|}\right|^{p} .
\end{gathered}
$$

Here the parameter $p$ is an exponent $\geq 1$ and is not the "pressure $p$ " in (1). Instead of varying $\kappa$ for the particular flow problem, one can vary $p$. For larger $p$, less numerical dissipation is added. Note that by varying $p \geq 1$ in (7), one can essentially increase the order of accuracy of the filter. The $\alpha_{j+\frac{i}{2}}^{l}$ are elements of $R_{j+\frac{1}{2}}^{-1}\left(U_{j+1, k}-U_{j, k}\right)$. The corresponding $\alpha_{j+\frac{1}{2}}^{l}, \phi_{j+\frac{1}{2}}^{l}$ and $R_{j+\frac{1}{2}}$ using the MLSCL formulation are instead functions of the left and right states of $U$.

Multi-Resolution Non-orthogonal Wavelet Sensors: To avoid the tuning of the arbitrary parameters $\kappa$ and/or $p$ in ( $\bar{i}$ ), one can replace $\kappa \theta_{j+\frac{1}{2}}^{l}$ by a wavelet sensor $\omega_{j+\frac{1}{2}}^{l}$ (Sjogreen \& Yee, 2000). With a proper choice of the wavelet basis function, we have a better control on the proper distribution of numerical dissipations leading to a more accurate simulation than the AC.I sensor. Wavelets were originally developed for feature extraction in image processing and for data compression. It is well known that the regularity of a function can be determined from its wavelet coefficients (Daubechies 1992, Mallat \& Zhong 1992) far better than from its Fourier coefficients. By computing wavelet coefficients (of a suitable wavelet basis function). we obtain very precise information about the regularity of the function in question. As of the 1990's, wavelets are a new class of basis functions that are finding use in analyzing and interpreting turbulence data from experiments. They also are used for analyzing the structure of turbulence from numerical data obtained from DNS or large eddy simulation (LES). See Farge (1992) and her later work, and Perrier et al. (1999). Recently, wavelets have been used for grid adaptation (Gerritsen \& Olsson) and to replace existing basis functions in constructing more accurate finite element methods. Here we utilize wavelets to adaptively control the amount of numerical dissipation that is inherent in standard high-resolution shockcapturing schemes. The resulting wavelet sensors are readily available as more desirable grid adaptation indicators than the commonly used grid adaptation indicators.

The wavelet sensor of Sjogreen \& Yee is obtained by computing the so called "Lipschitz Exponent" of a chosen vector to be sensed with a suitable multi-resolution non-orthogonal wavelet basis function that is capable of detecting shocks, shears, spurious oscillations and turbulence. Here, "vectors or variables to be sensed" means the represented vectors or variables that are suitable for the extraction of the desired flow physics. The study in Sjogreen \& Yee showed that for a proper choice of the wavelet basis function, the wavelet sensor is physical problem-independent for all of the test cases considered in Yee et al. (1999a,b). The variables to be sensed can be the density and/or pressure, the characteristic variables, the $\alpha_{j+\frac{1}{2}}^{l}$, or the entropy variables $W$. There are two types of non-orthogonal wavelet basis functions that Sjogreen \& Yee considered. One is similar to the B-spline wavelet (Mallat \& Zhong) used by Gerritsen \& Olsson for grid adaptation and the other is modification of the multi-resolution method of Harten (1995) as a redundant multi-resolution wavelet. The B-spline wavelet sensor requires slightly more arithmetic operations than the redundant form of Harten wavelet sensor. The final form for $\omega_{j+\frac{1}{2}}^{l}$ involves mainly nested difference operators and least squares fits. However, the theory is quite involved. The reader is referred to Sjogreen and Yee for the exact 
formula and the references cited for background. It is noted that the dual purpose wavelet sensors (dynamic numerical dissipation controls and grid adaptation indicators) can be a stand alone option for a variety of schemes other than what is discussed here.

It is emphasized here that neither ACM nor wavelet sensors will be able to improve the accuracy at the shock and shear locations over the inherent shock-capturing capability of the nonlinear dissipation. The accuracy of the shock and shear is dictated by the chosen nonlinear dissipation. The role of the sensors is to allow the full amount of numerical dissipation in shock and shear regions, and to limit the amount of numerical dissipation in regions immediately away from shock and shear locations and the rest of the flow field. Therefore, with a suitable sensor, one does not have to use CPU-intensive high order high-resolution shock-capturing numerical dissipation. since this type of dissipation generally gives a slightly more accurate solution away from discontinuities but exhibits similar shock and shear resolutions as second or third-order high-resolution numerical dissipations.

Full Discretizations: If a multistage time discretization such as the Runge-Kutta method is desired, the high order non-dissipative spatial differencing base scheme is applied at every stage of the Runge-Kutta method. If viscous terms are present, they use the same order and type of base scheme as for the inviscid terms. There are two methods for applying the characteristic filter. Method 1 is to apply the filter at every stage of the Runge-Kutta step. Method 2 is to apply the filter at the end of the full Runge-Kutta step. For inviscid and strong shock interactions, method 1 might be more stable.

If one desires a time discretization that belongs to the class of linear multistep methods (LMMs), e.g.. trapezoidal rule or three-point backward differentiation, then the filter can be applied as a numerical dissipation vector in conjunction with the base scheme. The filter in this case is evaluated at $U^{n}$ for explicit LMMs. For implicit. LMMs additional similar filters evaluated at the $n+1$ time level might be involved. Alternatively, method 2 can be applied to LMMs as well. In this case, we apply the filter after the completion of the implicit time step.

As an example, we illustrate the complete form of the schemes for Runge-Kutta methods with the filters applied at the completion of a full Runge-Kutta time step. Let $\widehat{U}^{n+1}$ be the solution after one full Runge-Kutta time step using a non-dissipative spatial base scheme. Note that if entropy splitting is employed, the base scheme is applied to the split form of the inviscid flux derivatives. Then the solution at the next time level $U^{n+1}$ is

$$
U_{j, k}^{n+1}=\widehat{U}_{j, k}^{n+1}-\frac{\Delta t}{\Delta x}\left[\widetilde{F}_{j+\frac{1}{2}, k}^{*}-\tilde{F}_{j-\frac{1}{2}, k}^{*}\right]+\frac{\Delta t}{\Delta y}\left[\widetilde{G}_{j, k+\frac{1}{2}}^{*}-\widetilde{G}_{j, k-\frac{1}{2}}^{*}\right]
$$

Here. $\tilde{F}_{j \pm \frac{1}{2}, k}^{*}$ and $\tilde{G}_{j, k \pm \frac{1}{2}}^{*}$ are evaluated at $\widehat{U}^{n+1}$.

\section{Numerical Examples}

We summarize the performance of this class of schemes by illustrating four perfect gas test cases with distinct flow properties. The first is inviscid and the last three are compressible full Navier-Stokes computations. The four test cases are: (1) a 2-D inviscid horizontally convecting vortex with periodic boundary conditions (BCs), (2) a 2-D vortex pairing in a time-developing mixing layer with shock waves formed around the vortices, (3) a 2-D shock wave impinging on a spatially evolving mixing layer where the evolving vortices must pass through a shock wave. which in turn is deformed by the vortex passage, and (4) a direct numerical simulation of a 3-D shock-free compressible turbulent plane channel flow. Figures 1-4 show the schematic, flow conditions. grid size and the computational domains of the four test cases. 
In all of the computations the classical fourth-order Runge-Kutta time discretization, and the non-compact central spatial interior schemes with the same order of accuracy and type of base scheme for the inviscid and viscous terms (if viscosities are present) are employed. If filters are used, they are applied at the end of the full Runge-Kutta time step. Roe's (1981) average states are used in (4), along with the Harten and Yee (Yee 1989, Yee et al. 1990) second-order upwind TVD dissipation portion for $\phi_{j+\frac{1}{2}}^{l}$ in (5). The parameters $p$ and $m$ are set to 1 and a small value of $10^{-6}$ is added to the denominator of (7) to avoid an extra logical statement for the ACM sensor. These will be notated as ACM or WAV (depending on whether $A C M$ or wavelet is used as sensor) with the following numbers indicating the order of the interior base scheme for the inviscid and viscous terms. For example, ACM66 (WAV66) means the use of sixth-order central as the base scheme for both the inviscid and viscous terms, and $A C M$ as sensor (wavelet as sensor). In order not to introduce additional notation, inviscid flow simulations are designated by the same notation, with the viscous terms not activated. Computations using entropy splitting are indicated by adding the letters "ENT" at the end as in ACM66-ENT (WAV66-ENT). Computation using $\mathcal{S}_{j+\frac{1}{2}}^{l}=1$, i.e, the sensor is turned off and the upwind TVD dissipation portion is used as the filter, will be notated by TVD as in TVD66. To examine the performance of the entropy splitting schemes where shock waves are absent. the computations also employ only the non-dissipative central base schemes (without the filters) designated as CEN66 and CEN66-ENT for sixth order unsplit and split forms of the inviscid flux derivatives respectively. The fifth-order WENO scheme of Shu (1997) will be notated as WENO5.

For the Navier-Stokes computations involving entropy splitting, the splitting is applied to the inviscid flux terms. and the symmetric form of the viscous flux is not used. Except for the periodic BCs and the 3-D channel problem, compatible stable boundary schemes of Strand, and Olsson are not used in order to study the benefit of the entropy splitting without considering the accompanying stable boundary schemes as a complete package for stability consideration. For the 3-D channel flow, a variant of the Strand boundary scheme developed by Carpenter et al. (1998) is employed. For this problem, we also reformulate the viscous term to minimize odd and even decoupling phenomena before the application of the central scheme (Sandham $\&$ Yee). For the second and third test cases, we lower the order of the base scheme near the boundary points for the boundary scheme. For the third test case, for simplicity, slip wall $\mathrm{BC}$ is used for the lower wall, and the upper $y$-direction physical $\mathrm{BC}$ is overspecified and nonreflecting $\mathrm{BC}$ is not used. The various ways of imposing physical and boundary schemes for the different cases illustrate the robustness of the schemes and are an indication of the added value of entropy splitting used in conjunction with interior and boundary schemes that do not satisfy the generalized energy estimate. In order to assess the true performance of the algorithm. no attempt is made to enhance the resolution using appropriate adaptive grid procedures.

Figures 5-16 show the following comparisons:

1. Numerical Dissipation Sensor Control vs. No Sensor: Comparisons of TVD66 and ACM66 for test cases 2 and 3 are shown in Figures 5 and 6. For test case 2, the ACM66 using a $41 \times 41$ grid produces a solution slightly more accurate (around the shear regions) than TVD66 using a $101 \times 101$ grid. Note that the widths of the shock resolution of ACM66 and TVD66 are the same as discussed in Section II (the paragraph before the full discretization subsection). For the TVD66, ACM66 and WAV66 comparison, results should be compared with Figures 12 and 13 a results. 
2. Entropy Splitting vs. Unsplit: Comparisons of CEN66 vs. CEN66-ENT, and ACM66 vs. ACM66-ENT for test case 1 are shown in Figures 7-10. The CEN66 diverged shortly after $t=55$ as opposed to over 6 times the integration time longer for CEN66-ENT with an almost perfect vortex preservation. For even longer time integrations, numerical dissipation is needed. The amount for ACM66-ENT is less than for ACM66. For all simulations involving entropy splitting for test case 1 , we set the splitting parameter $\beta=1$ and $p^{*}$ in (3) to be a constant (for isentropic flow). It seems that ACM66-ENT requires a factor of at least $\frac{\beta}{1+\beta}$ less numerical dissipation over the ACM66. This factor is precisely the conservative portion of the entropy splitting of the inviscid flux derivatives. This factor is, however. greatly reduced if high shear or shock waves are present.

3. Performance of the 5th-Order WENO Scheme: We compare WENO5 with ACM66 for test cases 3 and 4 , and various mixing layer problems, including hypersonic speed. In all cases. WENO5 is more diffusive than ACM66. Figure 11 shows their comparison for test case 3, where the normalized temperature contours (top two) and Mach contours (bottom two) at $t=113.16$ on a $320 \times 81$ grid for WENO5 and a $321 \times 81$ grid for ACM66 are shown. The vortices are more diffusive in the WENO5 computations. There is a minor difference on the two simulations. The WENO5 code has a built-in nonreflecting $\mathrm{BC}$ on the upper $y$-direction. It is too costly to fully compare the WENO5 with the $3-\mathrm{D}$ channel DNS computations where a fully developed turbulence statistics grid refinement study was illustrated in Sandham \& Yee (2000) using CEN44-ENT. For WENO5, a smaller CFL number is used for stability. We compared the CPU time per time step and accuracy in a loose sense with CEN 14 -ENT. We use the solution at $t=150$ from the CEN44-ENT simulation as the starting solution for WENO5 and integrate for a time length of 20 and compare the turbulence statistics with the CEN44-ENT simulation. Our preliminary study shows that WENO5 costs a factor of six more CPU time with less accurate turbulence statistics than CEN44-ENT. See Hadjadj et al. (2001) for details.

4. ACM sensor vs. Wavelet sensor: Sample computations using WAV66 for test cases 2 and 3 are shown in Figures 12-11. The accuracy of the two wavelet sensors, B-spline wavelet (WAV66-BS) or the redundant form of Harten wavelet (WAV66-RH) for test cases 1-3 (results not shown) is very similar and the effect on accuracy of the choice of the physical vector (density and/or pressure, characteristic variables, $\alpha_{j+\frac{1}{2}}^{l}$, or entropy variables $W$ ) to be sensed is not pronounced. In all cases, no physical problem-dependent parameter has to be tuned. The accuracy compared very well with that of the corresponding best tuned $\kappa$ for the individual test cases 1-3. In particular, the same accuracy was sustained, using WAV66-ENT for either the B-spline or the redundant form of Harten wavelet sensors for long time integrations of the vortex convection problems. as ACM66-ENT using $\kappa=0.01$ and $\Delta t=0.01$. Figure 12 shows the test case 2 results which should be compared with the ACM 166 results in Figure 5. Figures 13 and 14 show the test case 3 results. Figure 13a shows the density and pressure contours computations by WAV66-RH using the density and pressure as the functions to be sensed (see Figure 6 for comparison with ACM66). Figure 13b shows the corresponding estimated Lipschitz exponent (alpha) for the density and pressure at $t=120$ and the wavelet sensor itself. Figure 14a shows the wavelet sensor applied to the density and pressure in the $x$ and $y$-directions, and the square root of the sum of these quantities in the $x$ and $y$-directions. Figure 14b shows the corresponding contours using the ACMI sensor. The wavelet sensor was able to extract the full feature of the flow structure far better than the ACM sensor. See Sjogreen and Yee for the exact formula and detailed numerical experiments. 
5. Fourth-Order Central Differencing with Entropy Splitting vs. Spectral Method: A grid refinement study of a compressible turbulent DNS and a comparison of CEN44-ENT with the incompressible simulation using a spectral method for test case 4 are shown in Figures 15 and 16. For the physical problems, background and grid refinement study, see Sandham \& Yee for details. These computations require very long time integrations of the full Navier-Stokes equations before fully developed turbulence statistics can be obtained. A very accurate data base is available for the 3-D DNS incompressible turbulence simulations. For comparison, we use a Mach number of 0.1 and a Reynolds number of 180 . Very accurate fully developed turbulence statistics were obtained using coarse to moderate grid sizes and without filters. The results compared well with the best spectral method of incompressible Lavier-Stokes flow formulations which may use de-aliasing, skew-symmetric formulation or energy conservation (e.g., variables on a staggered grid) to obtain a robust method. The angle brackets $\langle>$ denote averages over the homogeneous spatial directions and time while double primes denote deviation from mass-weighted (Favre) averages.

\section{Concluding Remarks}

In addition to the systematic theoretical development of simple parallelizable nonlinear stable schemes for IBVPs, their extension and their abilities to accurately simulate a wide range of physical applications for the Euler and Navier-Stokes equations, there are two key items that might have potential impact on a wide range of complex practical computations. One is the possible applicability of entropy splitting beyond the boundary of the theory indicated and the other is the wavelet sensors.

The numerical results indicate that entropy splitting alone can improve nonlinear stability even when one employs boundary schemes that do not satisfy the discrete generalized energy estimate. This stability property is valuable not just to the class of schemes in question, but might also be applicable to other schemes commonly used in practical CFD applications. This finding actually is not surprising since one should, if possible, condition the governing equations before the application of a suitable numerical method that can handle the type of physics inherent in the physical model.

The proposed wavelet sensors. unlike the ACM sensor, can detect most of the distinct flow features, including turbulence, leading to an automatic selection of the appropriate distribution of numerical dissipation. In addition. these wavelet sensors are free of physical problerndependent parameters for the three test cases, and they are also good grid adaptation indicators (Gerritsen \& Olsson) when compared to the ones commonly used in practice. Consequently, a new dual purpose adaptive method is readily available leading to dynamic numerical dissipation controls and improved grid adaptation indicators. This dual purpose adaptive method can also serve as a stand alone option for other numerical schemes. These are subjects of ongoing research.

\section{Acknowledgment}

Special thanks to Marcel Vinokur and Dennis Jespersen for their critical review of the manuscript. This work has been supported in part by RIACS of NASA Ames Research Center.

\section{References}

M.H. Carpenter, J. Nordstrom and D. Gottlieb (1998), "A Stable and Conservative Interface Treatment of Arbitrary Spatial Accuracy," NASA/CR-1998-206921 (also ICASE Report 9812). 
I. Daubechies (1992). Ten Lectures on Wavelets, CBMS-NSF Regional Conference Series in Applied Mathematics. No 61. SIAM.

M. Farge (1992), "Wavelet Transforms and their Applications to Turbulence," Ann. Rev. of Fluid Mech., 24, 395-457.

A. Hadjadj, H.C. Yee and N.D. Sandham (2001), "Comparison of WENO with Low Dissipative High Order Schemes for Compressible Turbulence Computations", RIACS Technical Report, NASA Ames Research Center.

M. Gerritsen and P. Olsson (1996), "Designing an Efficient Solution Strategy for Fluid Flows: I. A Stable High Order Finite Difference Scheme and Sharp Shock Resolution for the Euler Equations," J. Comput. Phys. 129, 245.

A. Harten (1978), "The Artificial Compression Method for Computation of Shocks and Contact Discontinuities: III. Self-Adjusting Hybrid Schemes," Math. Comp. 32, 363.

A. Harten (1983), "On the Symmetric Form of Systems for Conservation Laws with Entropy," ICASE Report 81-34. NASA Langley Research Center, 1981 also, J. Comput. Phys. 49, 151.

A. Harten (1995), "Wultiresolution Algorithms for the Numerical Solution of Hyperbolic Conservation Laws." Comm. Pure Appl. Math., 48, 1305-1342

S. Mallat and S. Zhong (1992), "Characterization of Signals from Multiscale Edges," IEEE Transactions on Pattern Analysis and Machine Intelligence, 14, 710-732.

P. Olsson, (1995) "Summation by Parts, Projections and Stability," Math. Comp. 64, 1035.

P. Olsson (1995). "Summation by Parts, Projections and Stability II.," Math. Comp. 64, 212.

P. Olsson (1995), "Summation by Parts, Projections and Stability III," RIACS Technical Report 95-06. NASA Ames Research Center.

P. Olsson and J. Oliger (199-1). "Energy and Maximum Norm Estimates for Nonlinear Conservation Laws," RI.ACS Technical Report 94-01, NASA Ames Research Center.

V. Perrier, T. Philipovitch and C. Basdevant (1999). "Wavelet Spectra Compared to Fourier Spectra," Publication of ENS. Paris.

P.L. Roe (1981). "Approximate Riemann Solvers, Parameter Vectors, and Difference Schemes," J. Comput. Phys. 43. 357.

N.D. Sandham, and H.C. Yee (2000), "Entropy Splitting for High Order Numerical Simulation of Compressible Turbulence," RIACS Technical Report 00.10, June 2000, NASA Ames Research Center; Proceedings of the First International Conference on CFD, July 10-14, 2000, Kyoto, Japan.

C.W. Shu. "Essentially Non-Oscillatory and Weighted Essentially Non-Oscillatory Schemes for Hyperbolic Conservation Laws." NASA/CR-97-206253, 1997.

B. Sjogreen. "Numerical Experiments with the Multiresolution Scheme for the Compressible Euler Equations," J. Comput. Phys., 117, 251-261 (1995).

B. Sjogreen and H.C. Yee (2000), "Wavelet Based Adaptive Numerical Dissipation Control for Shock-Turbulence Computations," RIACS Technical Report, NASA Ames Research Center.

J.L. Steger and R.F. Warming. (1981) "Flux Vector Splitting of Inviscid Gas Dynamics Equations with Applications to Finite Difference Methods," J. Comput. Phys. 40, 263.

B. Strand (1994), "Summation by Parts for Finite Difference Approximations for $d / d x$," J. Comput. Phys. 110. 47.

M. Vinokur and H.C. Yee, (2000) "Extension of Efficient Low Dissipative High Order Schemes for 3-D Curvilinear Moving Girds," NASA Technical Memorandum 209598, June 2000, NASA 
Ames Research Center; Proceedings of Computing the Future III: Frontiers of CFD - 2000, June 26-28, 2000, Half Moon Bay, CA

H.C. Yee (1989), "A Class of High-Resolution Explicit and Implicit Shock-Capturing Methods," VKI Lecture Series 1989-04 March 6-10, 1989, also NASA TM-101088, Feb. 1989.

H.C. Yee, G.H. Klopfer and J.-L. Montagne (1990), "High-Resolution Shock-Capturing Schemes for Inviscid and Viscous Hypersonic Flows," J. Comput. Phys., 88, 31.

H.C. Yee, N.D. Sandham and M.J. Djomehri (1999a), "Low Dissipative High Order ShockCapturing Methods Using Characteristic-Based Filters," RIACS Technical Report 98.11, May 1998, also, J. Comput. Physics, 150, 199-238.

H.C. Yee, M. Vinokur and M.J. Djomehri (1999b), "Entropy Splitting and Numerical Dissipation," NASA Technical Memorandum 208793, August, 1999, NASA Ames Research Center.

H.C. Yee, M. Vinokur and M.J. Djomehri (2000), "Entropy Splitting and Numerical Dissipation," J. Comput. Phys., 161. 1-49, 2000. 


\section{Tables Captions}

Table 1. Flow Chart of the efficient low dissipative high order schemes.

Figures Captions

Fig. 1

Fig. 2

Fig. 3

Fig. 4

Fig. 5. Vortex pairing: Comparison of ACM66 with TVD66 $\left(\kappa \theta_{j+\frac{1}{2}}^{l}=1\right)$ with reference solution ACM 44 (201 $\times 201$ grid), illustrated by the normalized temperature contours at time $t=160$ on $101 \times 101$ and $41 \times 41$ grids with $\kappa=0.7$ for the nonlinear fields and $\kappa=0.35$ for the linear fields.

Fig. 6. Shock-shear layer interaction: Comparison of ACM66 with TVD66 $\left(\kappa \theta_{j+\frac{1}{2}}^{l}=1\right)$ with reference solution $\mathrm{ACM} 44(641 \times 161$ grid $)$, illustrated by the density contours at $t=120$ on a $321 \times 81$ grid with $\kappa=0.35$ for the nonlinear fields and $\kappa=0.175$ for the linear fields.

Fig. 7. Convecting Vortex: Comparison of CEN66 with the exact solution (I.C.), illustrated by density contours at $t=20,30,40,50,55$ for $\Delta t=0.01$ on a $80 \times 79$ grid.

Fig. 8. Conrecting Vortex: Comparison of CEN66-ENT with the exact solution (I.C.). illustrated by density contours at $t=100,200,300,400,500$ for $\Delta t=0.01$ on a $80 \times 79$ grid

Fig. 9. Convecting Vortex: Comparison of ACM66 with the exact solution (I.C.), illustrated by density contours at $t=100,200,300,400,500,600,700,800,900,1000,1100$ for $\Delta t=0.04$ and $\kappa=0.04$ on a $80 \times 79$ grid.

Fig. 10. Convecting Vortex: Comparison of ACM66-ENT with the exact solution (I.C.), illustrated by density contours at $t=100,200,300,400,500,600,700,800,900,1000,1100$ for $\Delta t=0.01$ and $s:=0.01$ on a $80 \times 79$ grid.

Fig. 11. Shock-shear layer interaction: Comparison of ACM66 with WENO5, illustrated by the normalized temperature contours (top two plots) and Mach contours (bottom two plots) at $t=113.16$ on a $321 \times 81$ grid for ACM66 with $\kappa=0.35$ for the nonlinear fields and $\kappa=0.175$ for the linear fields, and a $320 \times 81$ for the WENO5.

Fig. 12. Vortex pairing: WAV66-RH computations, illustrated by the density and normalized temperature contours at time $t=160$ on a $101 \times 101$ grid using the density and pressure as functions to be sensed.

Fig. 13. Shock-shear layer interaction: WAV66-RH computations illustrated by (a) density and pressure contours (top 2 plots), and (b) estimated Lipschitz exponent $\alpha$ and the wavelet sensor itself (bottom 2 plots) using the density and pressure as functions to be sensed at $t=120$ on a $321 \times 81$ grid.

Fig. 14. Shock-shear layer interaction: Comparison of ACM66 with WAV66 at $t=120$ on a $321 \times 31$ grid with $\kappa=0.35$ for the nonlinear fields and $\kappa=0.175$ for the linear fields for ACM66. illustrated by (a) contours of the sensor used by WAV66-RH applied to the density and pressure in the $x$ and $y$-directions, and the square root of the sum of these quantity (top 3 plots), and (b) the corresponding contours using the ACM sensor (bottom 3 plots).

Fig. 15. 3-D channel: Effect of grid refinement of a compressible turbulent DNS on (a) root 
mean square of $u, v$ and $w$ and (b) Reynolds stress and total stress, using CEN44-ENT and Carpenter et al. boundary scheme.

Fig. 16. 3-D channel: Comparison of root mean square of $u, v$, and $w$ on a $30 \times 30 \times 101$ grid of a compressible turbulent DNS using CEN44-ENT and Carpenter et al. boundary scheme with an incompressible turbulent DNS result on a $32 \times 32 \times 81$ grid using spectral method. 


\section{Efficient Low Dissipative High Order Schemes}

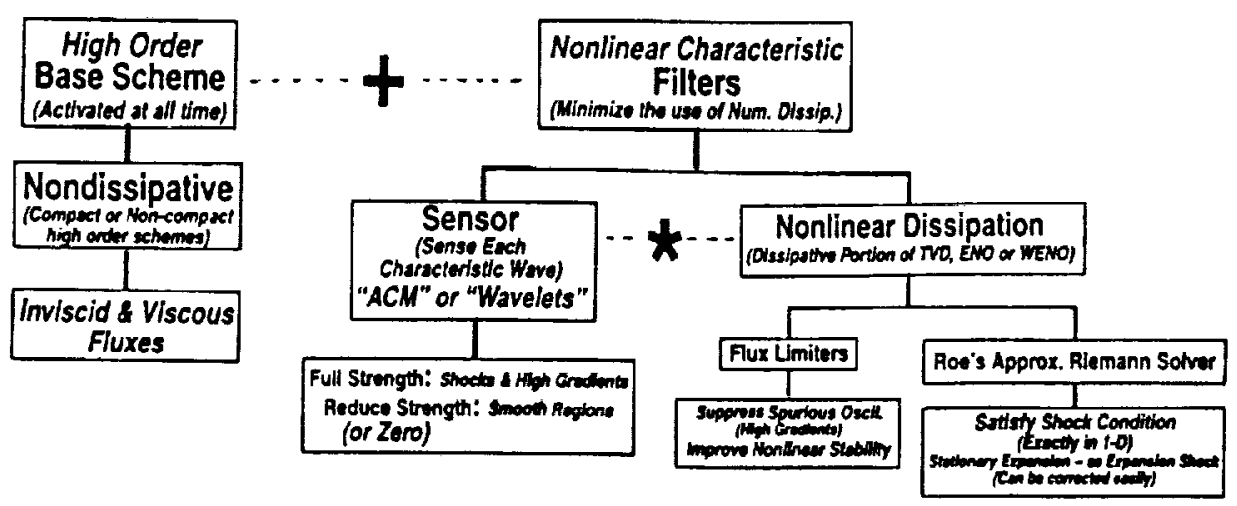

Apply schemes to the "Entrogy Solit Form" of the Flux Derivatives (Compare with Un-splif apprasech $-\beta=\infty$ )

$F_{0}=\frac{\beta}{\beta+1} \underbrace{F_{0}+\frac{1}{\beta+1} F_{W} W_{0}}_{i=0}$

Table 1. Flow Chart of the efficient low dissipative high order schemes.

\section{Isentropic Vortex Evolution \\ (Horizontally Convecting Vortex, vortex strength $\beta=5$ )}

Freestream:

$$
\left(u_{\infty}, v_{\infty}\right)=(1,0) ; P_{\infty}=\rho_{\infty}=1
$$

IC: Perturbations are added to the Ireestrean (not in entropy)

$$
\begin{aligned}
(\delta u, \delta v) & =\frac{\beta}{2 \pi} e^{2 \frac{1}{\gamma}}\left(-\left(y-y_{0}\right),\left(z-z_{0}\right)\right) \\
\delta T & =-\frac{(\gamma-1) \hat{\beta}^{2}}{8 \gamma \pi^{2}} e^{1-r^{2}}, \\
r & =\left(x-x_{0}\right)^{2}+\left(y-y_{0}\right)^{2}
\end{aligned}
$$

Computational Domain \& Grid Size:

$$
\begin{aligned}
& 0 \leq a \leq 10 \&-5 \leq y \leq 5 \\
& 80 \times 79 \text { Unliom grld } \\
& \text { Periodic BC in \& \& } y
\end{aligned}
$$

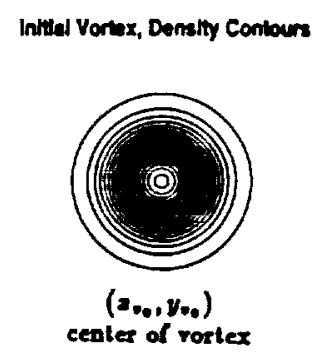

Fig. 1 


\section{Vortex Pairing in a Time-Developing Mixing Layer}

$$
\left(M_{c}=0.8, \text { Re }=1000, T_{R}=300 \mathrm{~K} \text {, Prandtl } \#=0.72\right)
$$

Normalized with vorticily thickness:

$$
\delta_{u}=\frac{u_{1}-u_{2}}{(d u / d y)_{m a}}
$$

T\& c: determined by assuming constant slagnation enthalpy

IC:

$u_{1}=1, u_{2}=-1, T_{1}=T_{2}$

Inital shear profilo: $\mathrm{U}=0.5 \tanh (2 \mathrm{y})$

Crocco-Busemann:

$$
c^{2}=c_{1}^{2}+\frac{\gamma-1}{2}\left(u_{i}^{2}-u^{2}\right)
$$

Initial perturbations:

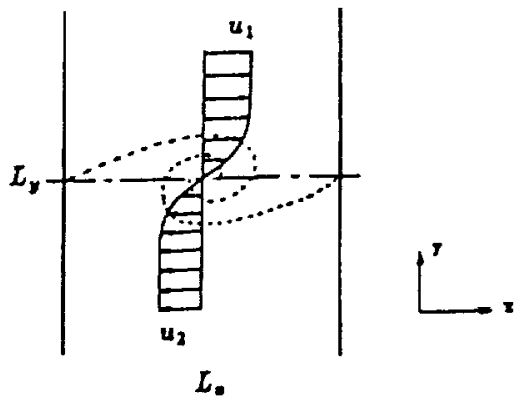

$$
\nu^{\prime}=\sum_{h=1}^{3} a_{h} \cos \left(2 \pi h z / L_{*}+\phi_{h}\right) e^{\left(-\nu^{\prime} / b\right)}
$$

$L_{.}=30, b=10$

$a_{1}=0.05, \phi_{2}=-\pi / 2$

$a_{1}=0.01, \phi_{1}=-\pi / 2$

BC: Periodic in $x$, slip walls in $y$

Grids:

$$
\begin{aligned}
& y=\frac{L_{y}}{2} \frac{\sinh \left(b_{y} \eta\right)}{\sinh \left(b_{y}\right)}, \quad L_{y}=100, b_{y}=3.4 \\
& \text { uniform in z }
\end{aligned}
$$

Fig. 2

\section{Shock Impingement on a Spatially-Developing Mixing Layer}

$$
\left(M_{c}=0.6, R e=500, T R=300 \mathrm{~K} \text {, Prandtl } \#=0.72 \text {, oblique shock angle }=12^{\circ}\right)
$$

$$
\begin{aligned}
& \text { Convective Mach I: } \quad M_{c}=\frac{u_{1}-u_{1}}{c_{1}+c_{2}} \\
& v^{\prime}=\sum_{h=1}^{3} a_{h} \cos \left(2 \pi k t / T+\phi_{h}\right) \exp \left(-y^{2} / b\right) \\
& a_{1}=0.05, \phi_{1}=0 \\
& a_{2}=0.05, \phi_{2}=\pi / 2
\end{aligned}
$$

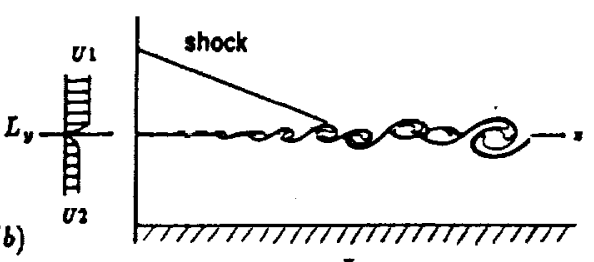

BC:

Obliqus shock wave forced by upper BC (impacts the shear layer at $x=90$ )

supersonic outtiow

lower boundary speclified as slip wall

Grlds:

$$
\begin{aligned}
& y=\frac{L_{y}}{2} \frac{\sinh (\eta)}{\sinh (1)}, \quad L_{*}=200, L_{y}=40 \\
& \text { uniform in }
\end{aligned}
$$

Fig. 3 


\section{DNS of 3-D Compressible Turbulent Channel Flow ( $M=0.1, R e=180$, Constant pressure gradient approach)}

Coleman, Kim \& Moser (1995): Uniform body lorce to drive the flow

$$
\phi=-\frac{1}{\rho} \frac{\partial P}{\partial x}
$$

Present Study: Constant pressure gradlent approach

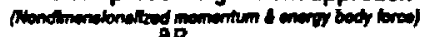

$-\frac{\partial P}{\partial x}=1$

$-v \frac{\partial P}{\partial x}=u$

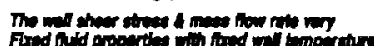

IC: Incompressible parabolic profile $\cdot \gamma^{+}$of 10
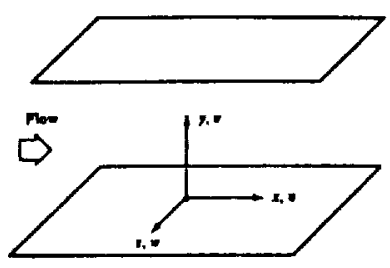

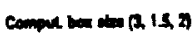

Rentines subloyor cloces to the wall a the log bow connectod to the center of the channel)

BC: No slip lsothermal wall

Perlodic in $x \& 2$

Grid sizes: $x \& 2$ uniform, y mildly stretched

$12 \times 12 \times 41$

$24 \times 24 \times 81$

$36 \times 30 \times 121$

$\mathrm{CF}=2.25$

$\frac{y}{h}=\frac{\tanh \left(c_{\eta} \eta\right)}{\tanh c_{\eta}}$

$\eta$ uniformly distributed on $[-1,1], c_{\eta}=1.7$

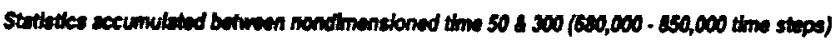

Entoor Soming Purandr. $\beta=4.0$

Fig. 4 


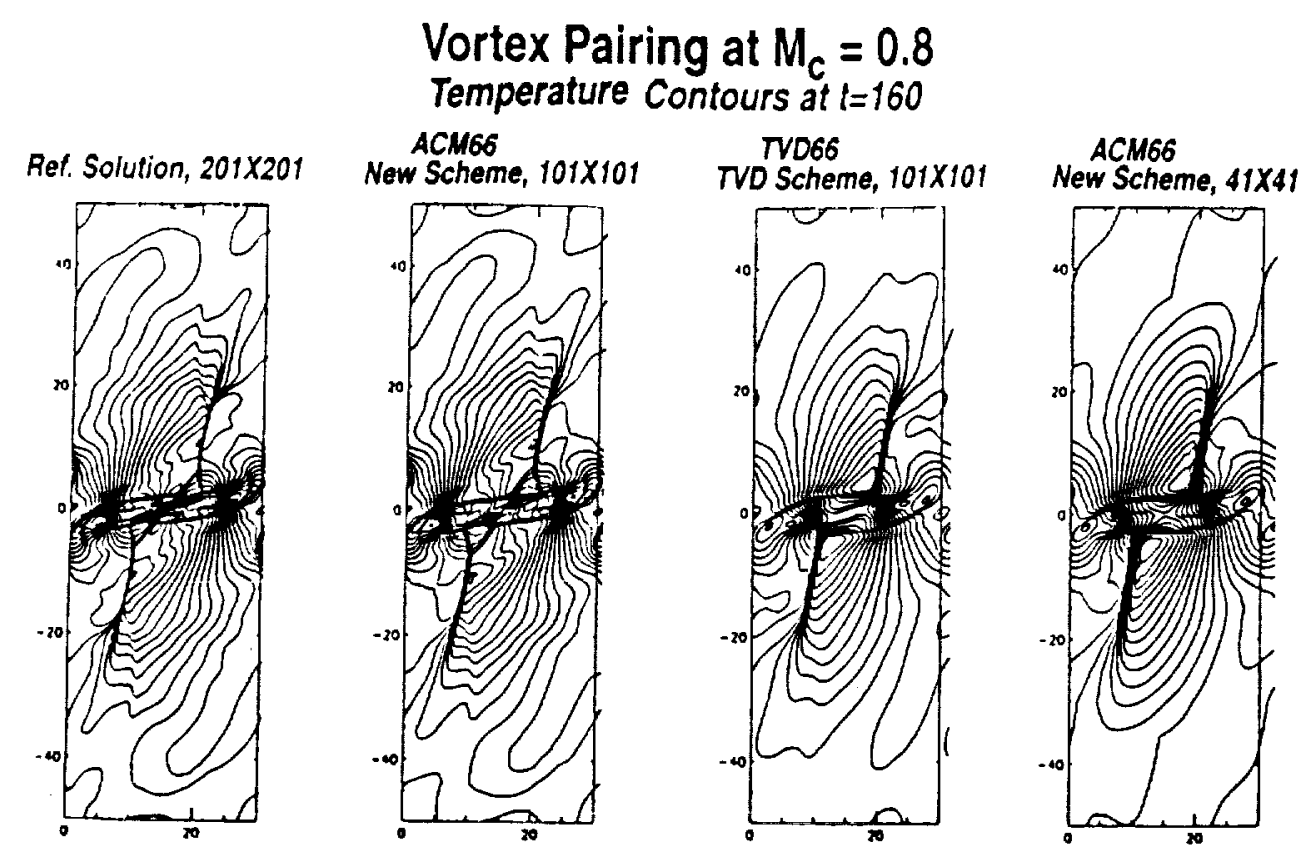

Fig. 5. Vortex pairing: Comparison of ACM66 with TVD66 $\left(\kappa \theta_{j+\frac{1}{2}}^{l}=1\right)$ with reference solution ACM44 (201 $\times 201$ grid), illustrated by the normalized temperature contours at time $t=160$ on $101 \times 101$ and $41 \times 41$ grids with $\kappa=0.7$ for the nonlinear fields and $\kappa=0.35$ for the linear fields.

\section{Shock Impingement on Mixing Layer Density Contours at $l=120$}

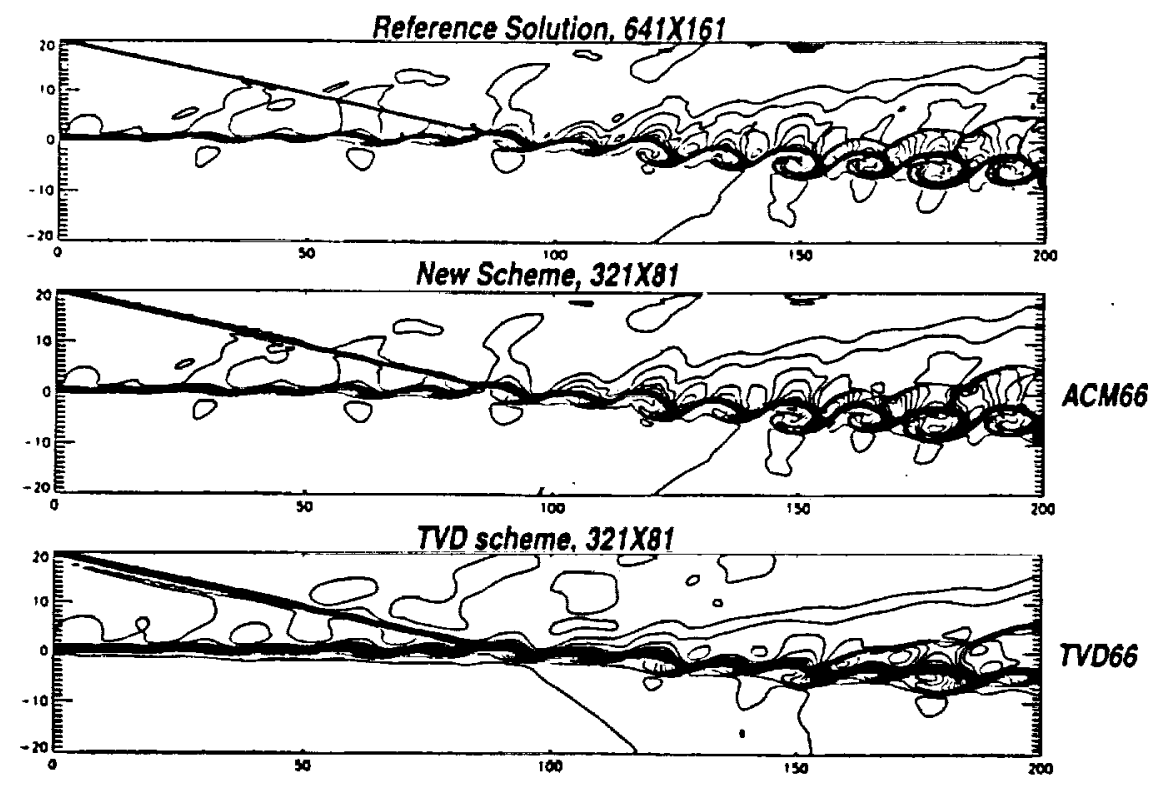

Fig. 6. Shock-shear layer interaction: Comparison of ACM66 with TVD66 $\left(\kappa \theta_{j+\frac{1}{2}}^{l}=1\right)$ with reference solution ACM44 $(641 \times 161$ grid), illustrated by the density contours at $t=120$ on a $321 \times 81$ grid with $\kappa=0.35$ for the nonlinear fields and $\kappa=0.175$ for the linear fields. 


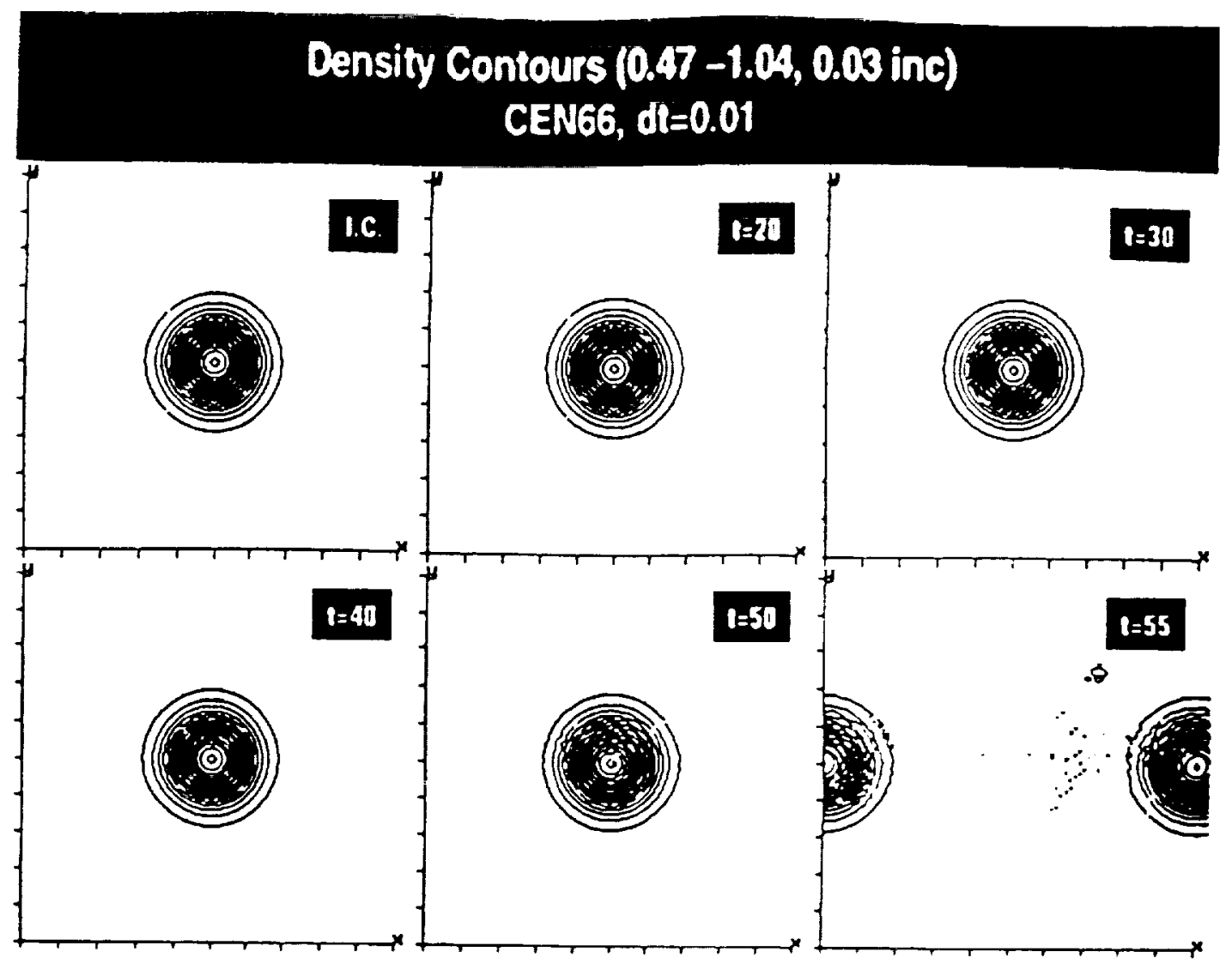

Fig. 7. Convecting Vortex: Comparison of CEN66 with the exact solution (I.C.), illustrated by density contours at $t=20,30,40,50,55$ for $\Delta t=0.01$ on a $80 \times 79$ grid.

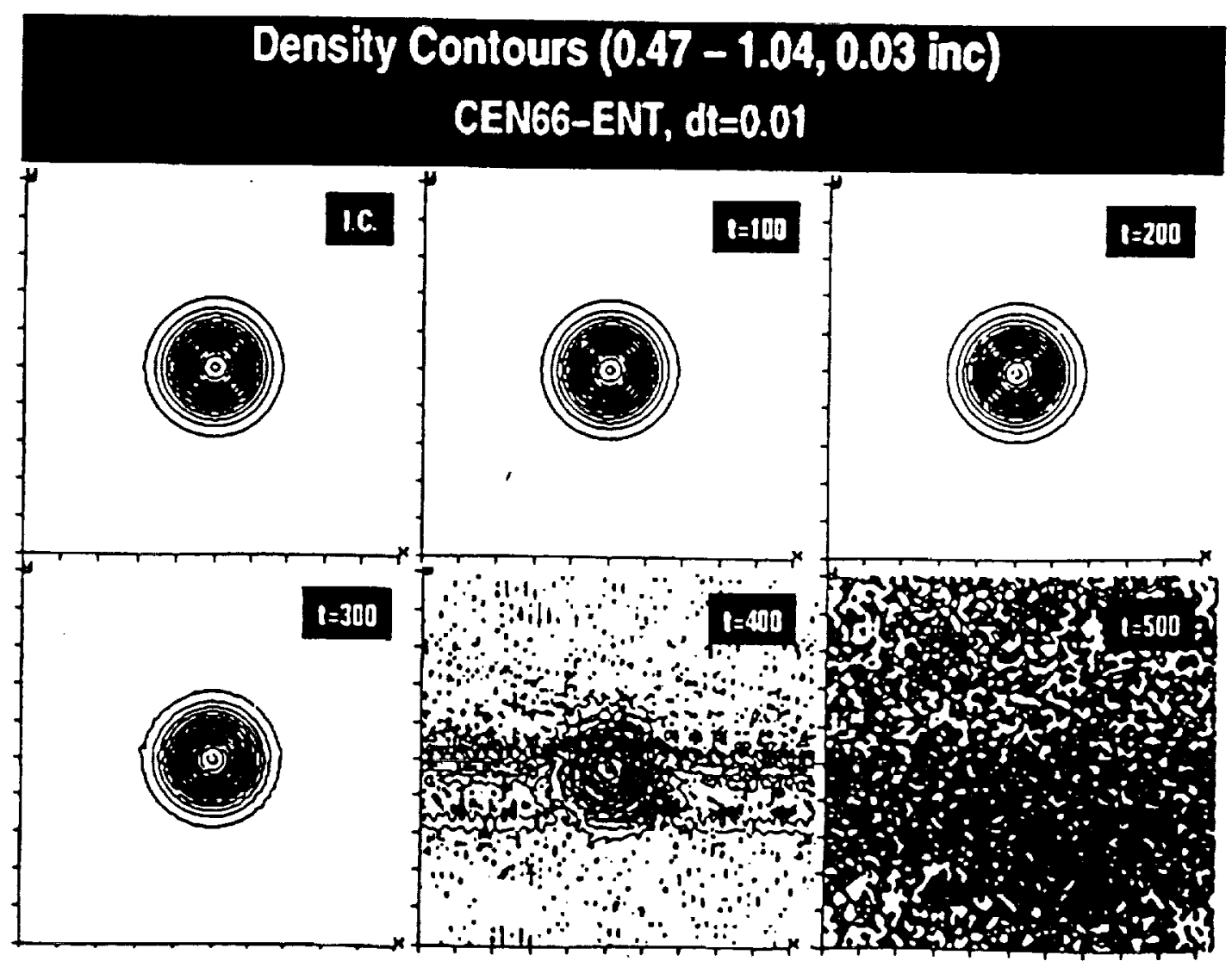

Fig. 8. Convecting Vortex: Comparison of CEN66-ENT with the exact solution (I.C.), illustrated by density contours at $t=100,200,300,400,500$ for $\Delta t=0.01$ on a $80 \times 79$ grid. 


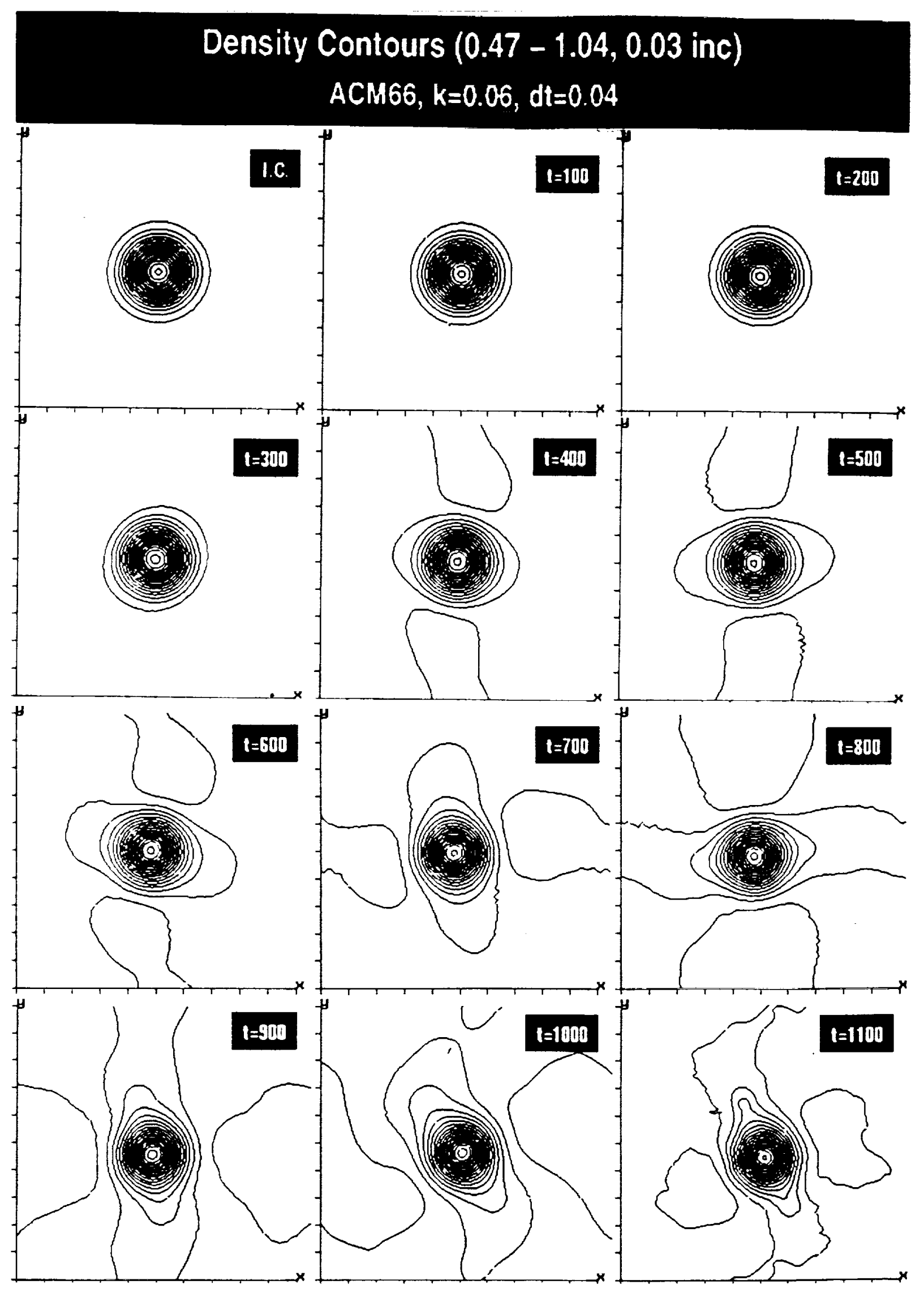

Fig. 9. Convecting Vortex: Comparison of ACM66 with the exact solution (I.C.), illustrated by density contours at $t=100,200,300,400,500,600,700,800,900,1000,1100$ for $\Delta t=0.04$ and $\kappa=0.04$ on a $80 \times 79$ grid. 


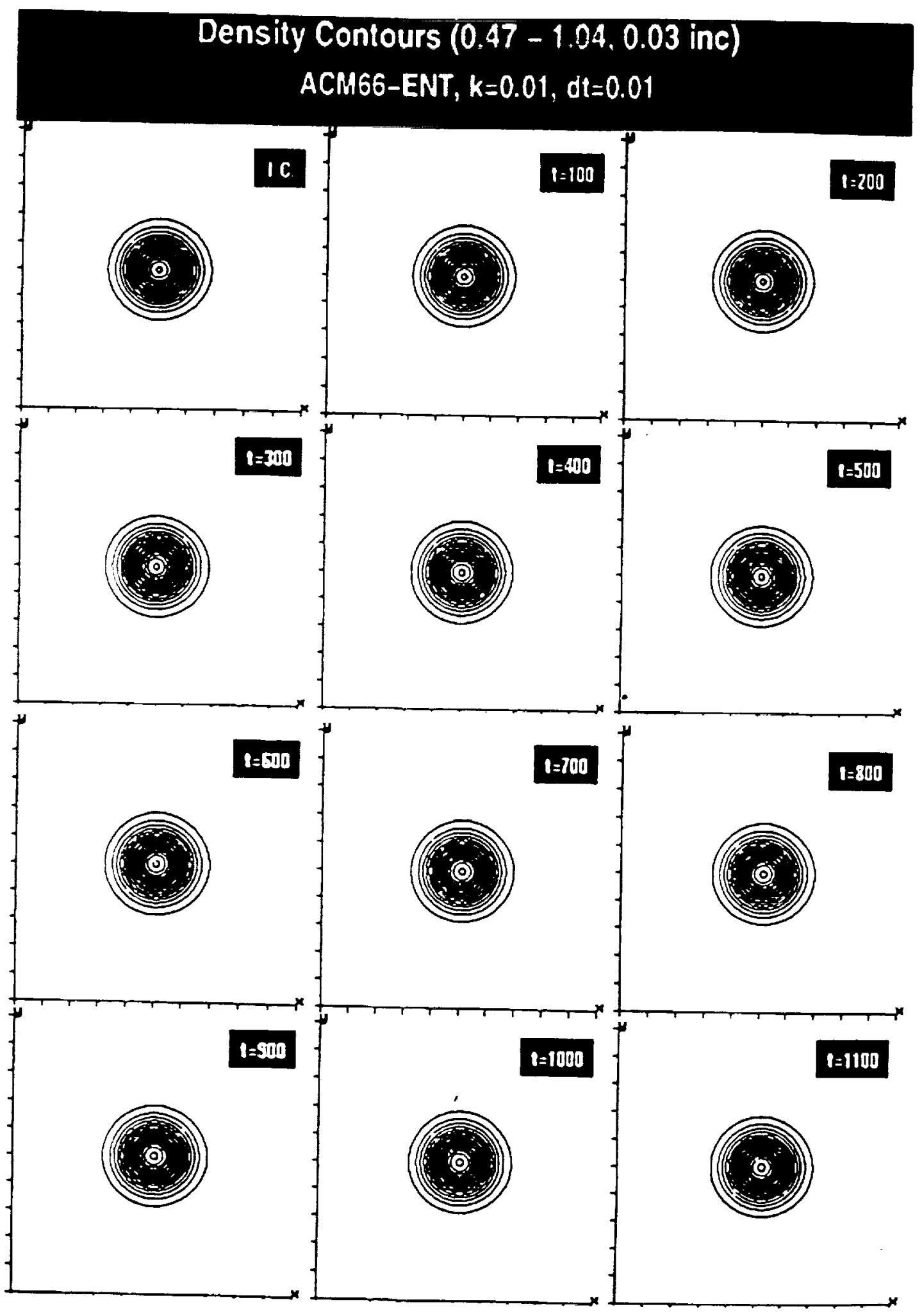

Fig. 10. Convecting Vortex: Comparison of ACM66-ENT with the exact solution (I.C.), illustrated by density contours at $t=100,200,300,400,500,600,700,800,900,1000,1100$ for $\Delta t=0.01$ and $\kappa=0.01$ on a $80 \times 79$ grid. 

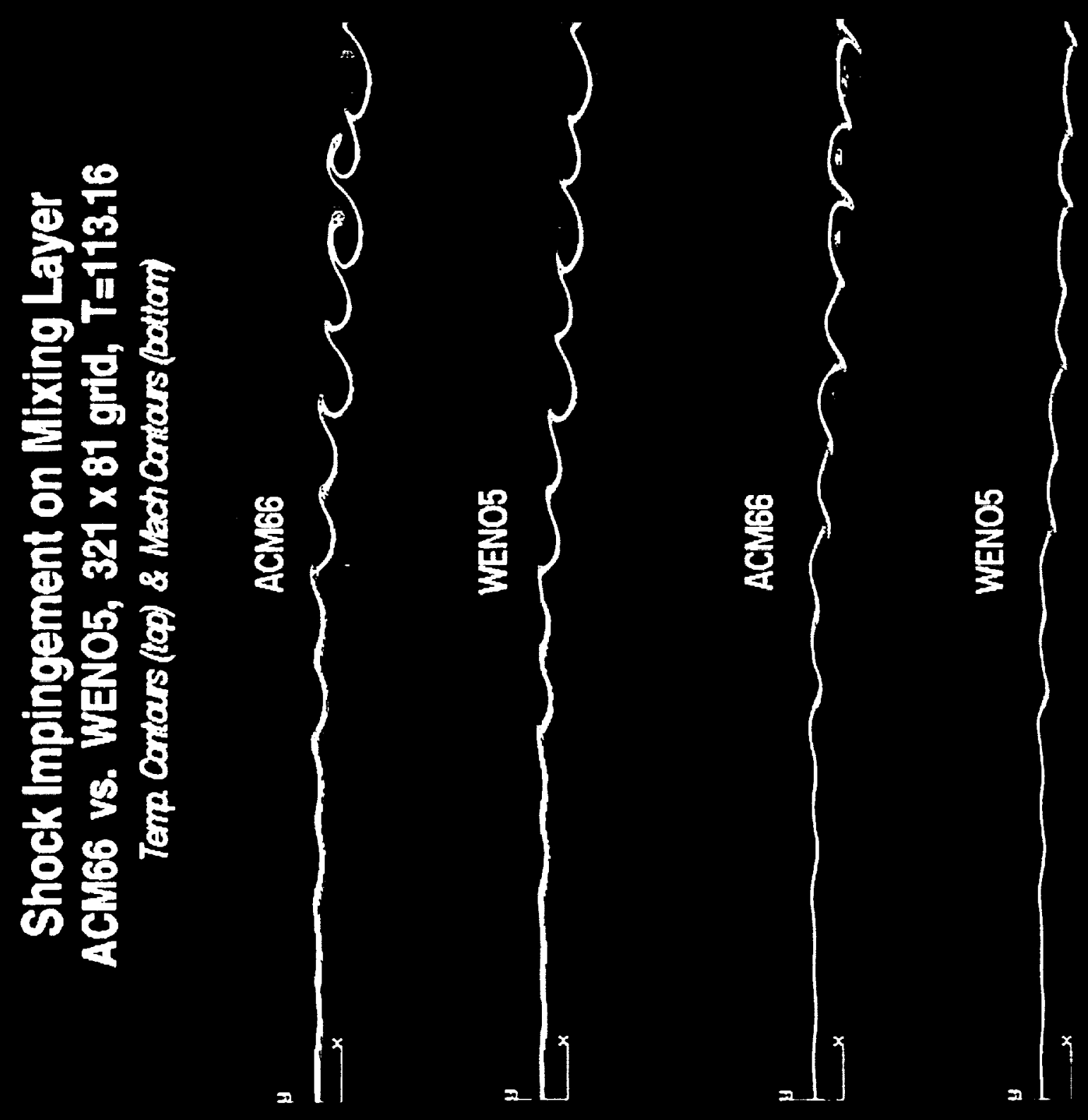

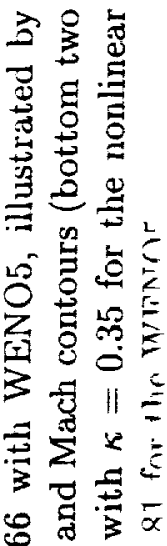

$\overbrace{0}$ त

$\sum_{4}^{0} \frac{0}{0} \sum_{0}^{0}$

范方=

ㅍㅎㅎ

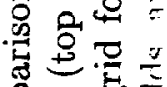

क 50

융

农 $x$

范

号 龸

焉要

吉

के

要

苞 夢

के है

渮:

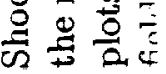

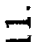

$\therefore$ 


\section{Vortex Pairing at $M_{C}=0.8$ WAV66-RH, $\mathrm{t}=160,101 \times 101$ grid}
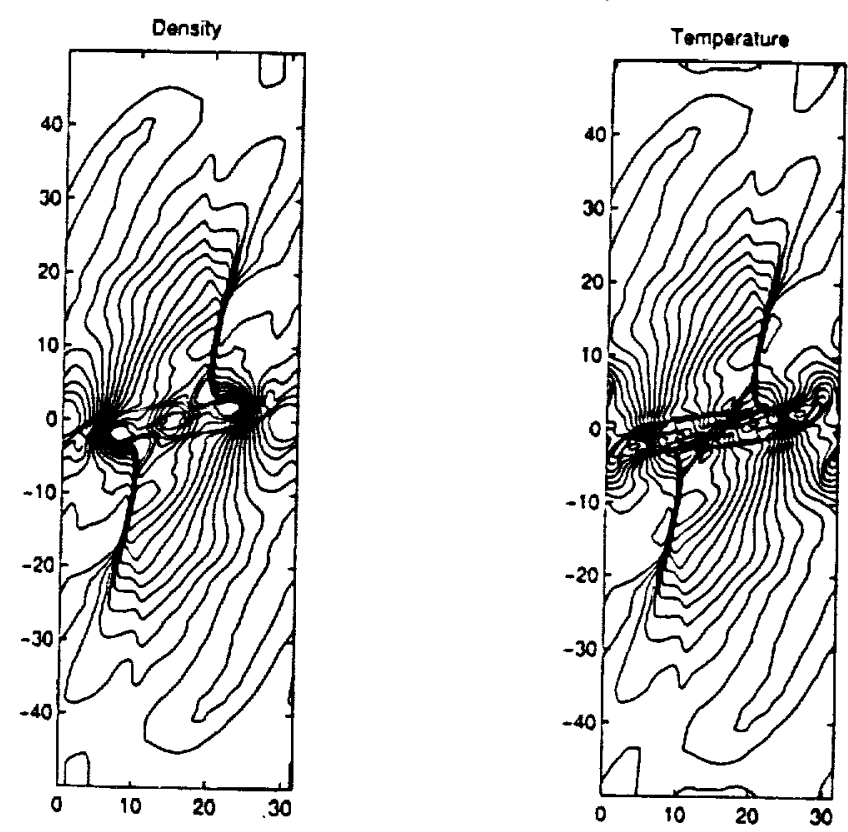

Fig. 12. Vortex pairing: WAV66-RH computations, illustrated by the density and normalized temperature contours at time $t=160$ on a $101 \times 101$ grid using the density and pressure as functions to be sensed. 


\section{Shock Impingement on Mixing Layer WAV66-RH, $\mathrm{t}=120,321 \times 81$ grid}
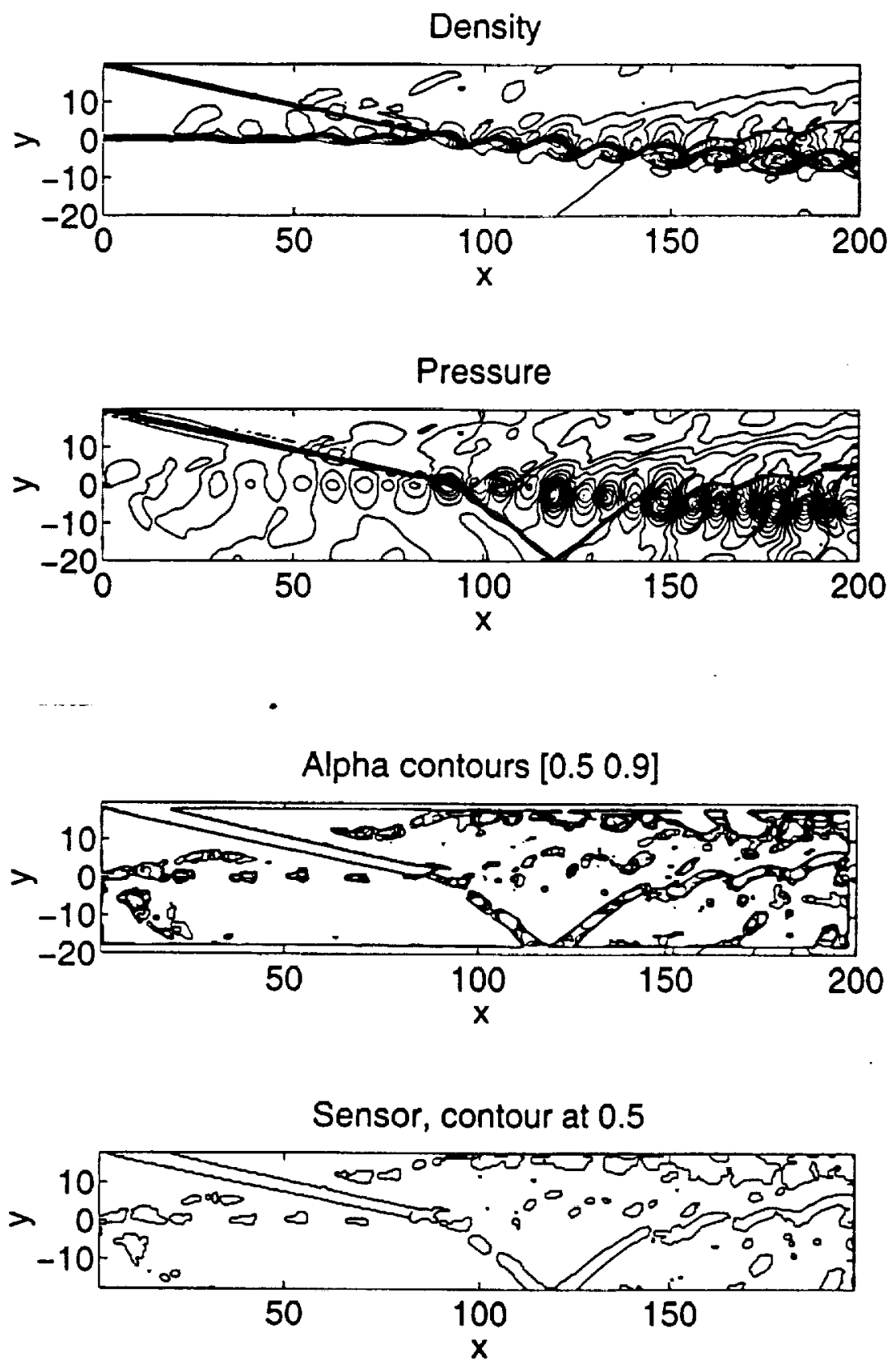

Fig. 13. Shock-shear layer interaction: WAV66-RH computations illustrated by (a) density and pressure contours, and (b) estimated Lipschitz exponent $\alpha$ and the wavelet sensor itself using the density and pressure as functions to be sensed at $t=120$ on a $321 \times 81$ grid. 


\section{Shock Impingement on Mixing Layer WAV66-RH, $t=120,321 \times 81$ grid}

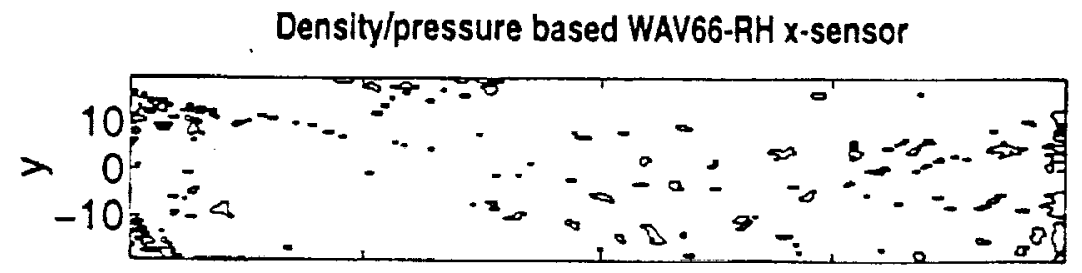

Density/pressure based WAV66-RH y-sensor

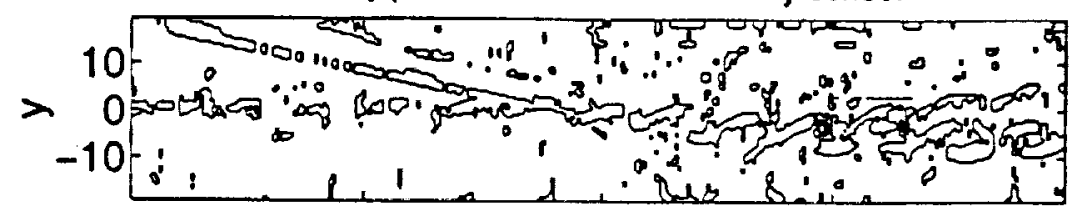

Density/pressure based WAV66-RH sqrt $\left(x^{2}+y^{2}\right)$

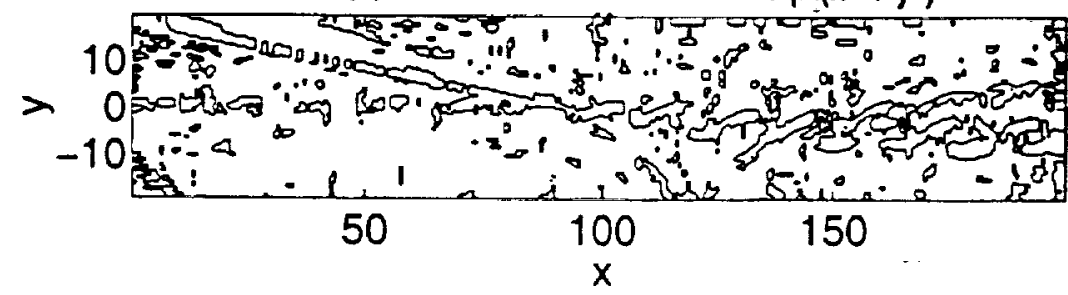

Density/pressure based ACM66-RH $x$-sensor

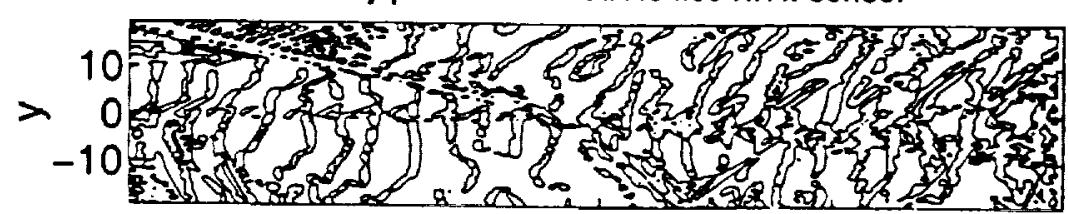

Density/pressure based ACM66-RH y-sensor
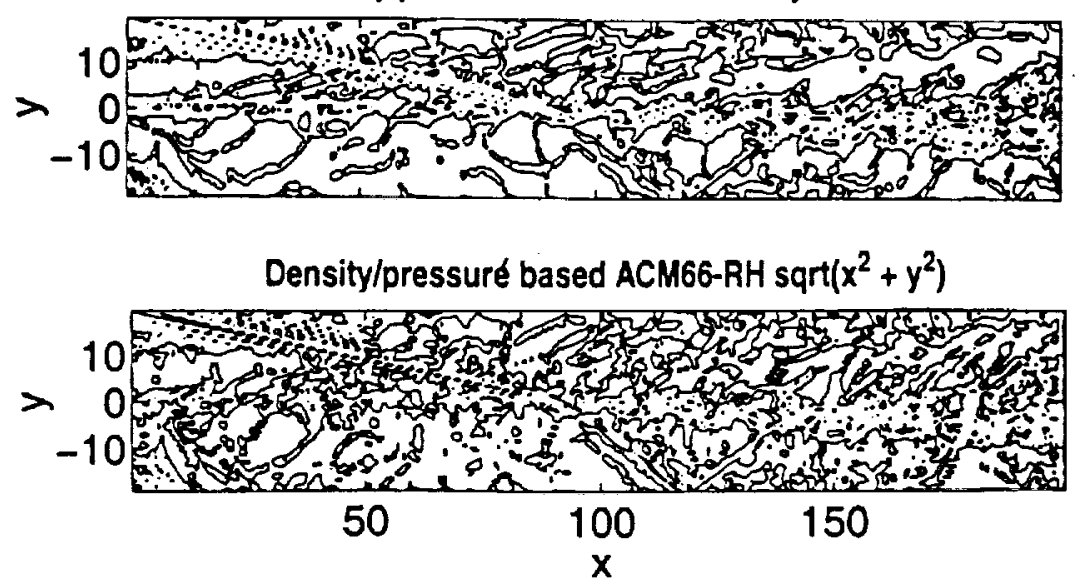

Fig. 14. Shock-shear layer interaction: Comparison of ACM66 with WAV66 at $t=120$ on a $321 \times 81$ grid with $\kappa=0.35$ for the nonlinear fields and $\kappa=0.175$ for the linear fields for ACM66, illustrated by (a) contours of the sensor used by WAV66-RH applied to the density and pressure in the $x$ and $y$-directions, and the square root of the sum of these quantity, and (b) the corresponding contours using the ACM sensor. 

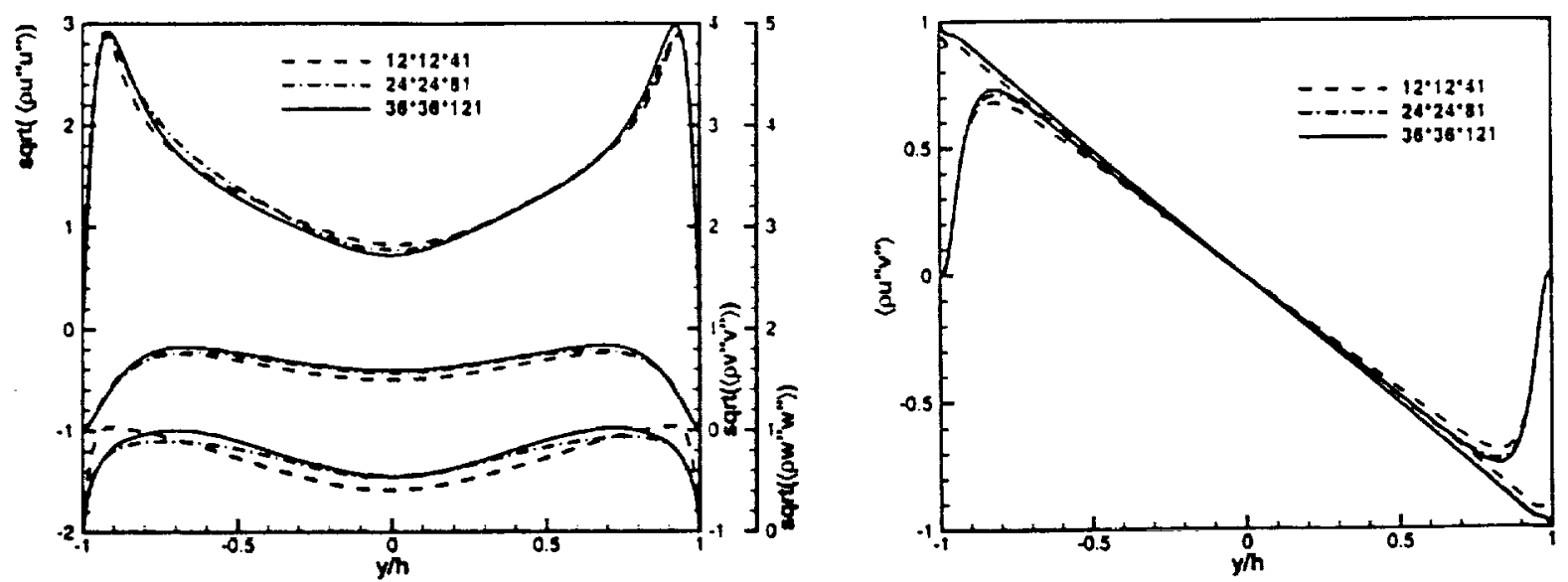

Fig. 15. 3-D channel: Effect of grid refinement of a compressible turbulent DNS on (a) root mean square of $u, v$ and $w$ and (b) Reynolds stress and total stress, using CEN44-ENT and Carpenter et al. boundary scheme.

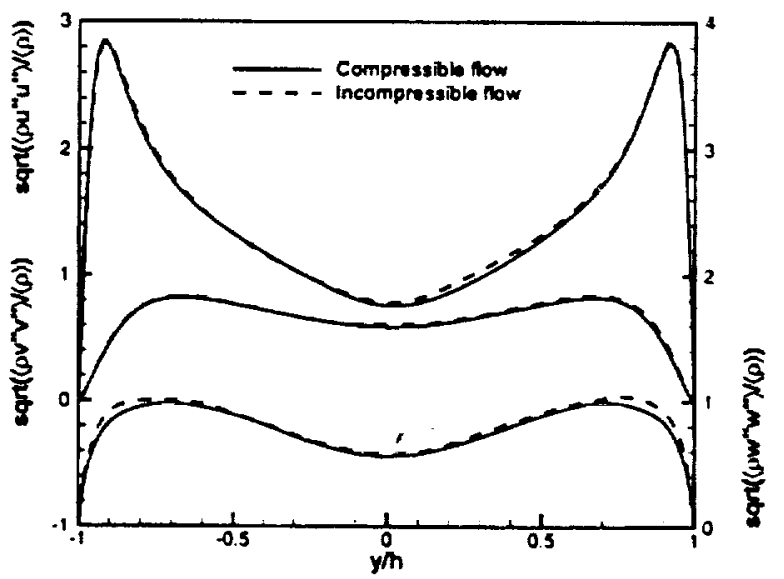

Fig. 16. 3-D channel: Comparison of root mean square of $u$, $v$, and $w$ on a $30 \times 30 \times 101$ grid of a compressible turbulent DNS using CEN44-ENT and Carpenter et al. boundary scheme with an incompressible turbulent DNS result on a $32 \times 32 \times 81$ grid using spectral method. 